\title{
Globalisation des marchés de capitaux et valorisation des actifs financiers
}

\author{
Dominique PEPIN ${ }^{*}$
}

Ce papier examine les effets de la libéralisation ou globalisation des marchés de capitaux sur les prix des actifs financiers. La comparaison des modèles de segmentation (douce) et d'intégration des marchés montre que la libéralisation des marchés a pour effet de favoriser la hausse des prix des actifs. Nous montrons que ce résultat ne résiste pas à une généralisation de l'analyse. La prise en compte du caractère multiple et simultanée des processus de libéralisation des marchés de capitaux amène à infirmer cette proposition, et celle selon laquelle l'amplification de la globalisation mène nécessairement à l'accroissement de l'intégration des marchés.

This paper examines changes in equities pricing following liberalization or globalization of capital markets. Comparing models of market (mild) segmentation with models of market integration shows that liberalization imply the rise in the equities prices. We show that this result does not hold in a more general model. By taking into account the manyfold and simultaneous feature of globalization process on capital markets, we can infirm both this proposition and the one which maintains that the more globalized are capital markets, the more integrated they are.

Plus un actif est risqué et plus sa plus rentabilité moyenne doit être importante. Cette compensation, sans laquelle les investisseurs qui éprouvent de l'aversion au risque refuseraient de détenir des actifs risqués, est le fondement de la théorie de l'évaluation des actifs financiers. Elle pose l'idée simple d'une «règle de proportion » entre risque et rentabilité, vérifiée à l'équilibre par tous les actifs échangés sur le marché.

\footnotetext{
${ }^{*}$ Centre de Recherche sur l'Intégration Economique et Financière, Université de Poitiers.
} 
Les premiers modèles d'évaluation (Sharpe [1964], Lintner [1965], Mossin [1966], Black [1972], Merton [1973], Ross [1976] et Lucas [1978]) furent développés dans un cadre dit domestique, ou sans référence particulière au marché international du capital. Les questions d'intérêt aux yeux des auteurs étaient alors purement techniques, et n'avaient trait qu'au pricing des actifs. Au fil des ans, est cependant apparu un débat portant sur le marché de référence des investisseurs. Doit-on considérer que l'évaluation des actifs dépend des marchés nationaux ; ou alors au contraire la reconnaissance grandissante de la globalisation des marchés amène-t-elle à considérer que le pricing des actifs doit s'établir sur une base internationale?

Aux premières modèles purement domestiques, succédèrent des analyses adhérant au contraire à l'hypothèse d'un pricing international des actifs, (Solnik ([1974], [1983]) et Adler et Dumas [1983]). Les conclusions tirées de ces modèles « internationaux », fondés sur l'hypothèse d'intégration $\mathrm{du}$ marché international $\mathrm{du}$ capital, ne sont pas fondamentalement différentes de celles des modèles domestiques : on observe toujours que la rentabilité moyenne d'un actif est à proportion du risque encouru. Cette règle de proportion entre risque et rentabilité est alors la même pour tous les actifs dans tous les pays, ce qui a amené de nombreux auteurs à caractériser l'intégration des marchés de capitaux par la validité de la loi du prix unique du risque (Stulz [1981a], Bordes [1988], Wheatley [1988], Chen et Knez [1995], Goyeau, Leonard et Pepin [1999]).

Cette situation idyllique de marchés parfaitement intégrés n'étant cependant pas plus conforme à la réalité que celle de marchés complètement segmentés, de nombreux auteurs ont corrigé les modèles internationaux en y incorporant l'existence de barrières à l'investissement international. Black [1974] et Stulz [1981b] incorporent des barrières fiscales, qui viennent distordre la règle de proportion entre rentabilité et risque. Errunza et Losq [1985], [1989], Eun et Janakiramanan [1986], Alexander, Eun et Janakiramanan [1987] et Hietala [1989] considèrent eux, non pas des barrières fiscales, mais des barrières d'accès qui discriminent les différents types d'investisseurs et les différents marchés, interdisant l'accès aux investisseurs de différents pays à certains segments du marché international des capitaux. Que l'on ait affaire à des barrières fiscales ou à des barrières d'accès, les implications empiriques ne changent guère (Alford [1993]), ce qui explique que 
l'on recourt toujours à un seul type de barrières pour modéliser la segmentation des marchés de capitaux.

Les barrières à l'investissement international s'étant progressivement atténuées au cours des deux dernières décennies, les implications de cette libéralisation ou globalisation des marchés de capitaux ont été naturellement étudiées ${ }^{1}$. Il ressort des analyses fondatrices de Errunza et Losq [1985] et de Alexander, Eun et Janakiramanan [1987] que la libéralisation des marchés de capitaux a pour effet non ambigu d'augmenter les prix des titres ou d'abaisser leur rentabilité attendue. En effet, à cause de la plus grande diversification des risques qui résulte de l'accès à un marché global du capital, les investisseurs peuvent réduire le risque non diversifiable de leur portefeuille. Cette réduction des risques s'accompagne alors d'une hausse de la demande d'actifs risqués, à laquelle le marché répond par une hausse des prix des actifs.

Des études empiriques récentes telles celles de Foerster et Karolyi [1999], Miller [1999], Bekaert et Harvey [2000], Doukas et Switzer [2000], Henry [2000] et Kim et Singal [2000] semblent corroborer cette assertion. Cependant, ces travaux ne sont pas exempts de critiques assez importantes, et leurs conclusions doivent être considérées avec précaution (Stulz [1999]). De surcroît, quand bien même on accepte leurs conclusions, Stulz [1999] fait remarquer que les effets de la globalisation sur le coût du capital ou sur les prix des actifs révélés par ces études n'ont pas l'ampleur attendue. A ses yeux cela constitue une énigme qu'il convient de résoudre.

Parmi les débuts d'explication de cette énigme donnés par Stulz [1999], l'une d'entre elles semble particulièrement pertinente. A l'inverse de ce qui est supposé dans les modèles théoriques, la globalisation ne se produit pas à l'issue d'une unique transformation, après laquelle tous les marchés seraient complètement intégrés. Elle se fait peu à peu, comme en témoigne la persistance du phénomène de home bias ${ }^{2}$. Au regard de ce phénomène, les conclusions théoriques d'un modèle comme celui de Errunza et Losq [1985] doivent être tempérées. Il faut tenir compte du home bias et du fait corrélatif que la libéralisation des marchés de capitaux n'a lieu que partiellement.

\footnotetext{
1 Pour désigner ce même phénomène d'atténuation ou de disparition complète des barrières à l'investissement international, certains auteurs parlent de libéralisation (Henry [2000], Bekaert et Harvey [2000]), d'autres de globalisation (Stulz [1999]).

${ }^{2}$ Voir Lewis [1999] pour une dicussion du biais domestique.
} 
Une autre limite très importante des modèles théoriques antérieurs est qu'ils considèrent une libéralisation « univariée ». Ils considèrent l'effet sur les prix de la libéralisation d'un seul marché, en faisant l'hypothèse restrictive du ceteris paribus habituel. En réalité, les libéralisations des différents marchés se produisent plus ou moins en même temps, et on peut s'interroger quants aux effets de cette simultanéité des processus de libéralisation sur le pricing des actifs financiers.

A cet effet, nous présentons ci-dessous un modèle moyenne-variance avec barrières d'accès, qui permet d'étudier de façon assez générale l'impact de l'atténuation des barrières à l'investissement international sur les prix des actifs financiers. Notre analyse de la libéralisation considère des situations de « départ» et $d$ ' " arrivée » quelconques ${ }^{1}$, ce qui conduit à dégager des résultats plus généraux que ceux de Errunza et Losq [1985]. Notre modèle a de surcroît l'avantage non négligeable de considérer une libéralisation simultanée des deux marchés considérés.

Nous montrons que la globalisation des marchés n'a pas un effet non ambigu sur les prix ou rentabilités attendues des actifs financiers. Le cadre plus général de notre modèle conduit à atténuer la force des conclusions tirées des modèles antérieurs, et plus encore permet de contester le fait que la levée des barrières à l'investissement dans un marché conduise toujours à l'augmentation des prix des actifs ${ }^{2}$. Ces résultats appuient aussi l'idée qu'une moindre ampleur des barrières n'a pas pour effet nécessaire de réduire les distorsions vis-à-vis de la loi du prix unique du risque. Il n'y a pas de raison que les écarts relatifs à la loi du prix unique du risque se résorbent de façon monotone au fur et à mesure que la libéralisation financière s'accentue. En d'autres termes, l'intégration des marchés de capitaux n'est pas nécessairement d'autant plus importante que les barrières à l'investissement sont moindres.

Dans une première partie, nous présentons la structure de notre modèle. Dans la deuxième, nous déduisons les valeurs d'équilibre des prix et des rentabilités attendues des actifs en fonction de l'intensité des barrières, ce qui nous permet dans une troisième et

\footnotetext{
${ }^{1}$ Nous considérons l'effet d'une globalisation partielle, dans le cadre de marchés qui auparavant ne sont pas forcément complètement segmentés, et qui n'amène pas obligatoirement les marchés à être complètement intégrés.

${ }^{2}$ Basak [1996] conteste aussi cette proposition.
} 
dernière partie d'étudier la valorisation des actifs financiers et les erreurs relatives à la loi du prix unique du risque en fonction de l'intensité de ces barrières.

\section{Présentation du modèle}

\subsection{Les hypothèses}

La struture de départ de notre modèle est très simple, ce qui n'empêche pas par ailleurs d'aboutir rapidement à des équations assez lourdes. Cette simplicité voulue constitue plus un avantage qu'un inconvénient, puisque qu'elle nous garde de la possibilité d'attribuer les résultats ambigus du modèle à la complexité de sa structure. Les conclusions que nous obtenons, à savoir que l'ouverture d'un marché ne conduit pas nécessairement à l'augmentation des prix des titres qui y sont échangés, et que les marchés de capitaux ne sont pas nécessairement d'autant plus intégrés que les barrières à l'investissement international sont moindres, nous apparaissent d'autant plus fortes que le modèle est le plus simple possible.

La représentation extrêmement élémentaire que nous donnons d'un hypothétique marché international du capital est la suivante :

(i) Deux pays, un actif risqué par pays. Par souci de simplicité, nous n'envisageons qu'un monde avec deux pays, les pays $A$ et $B$, ce qui suffit pour étudier les effets de l'imposition et de la levée de barrières à l'investissement international. Nous supposons qu'il existe un actif risqué par pays, en conséquence représentatif du marché. La diversité des échanges internationaux d'actifs risqués est donc aussi réduite que possible. Bien entendu, nous supposons que ces deux actifs ne sont pas identiques, pour laisser son intérêt à l'échange international. Les ventes à découvert sont autorisées, de sorte que l'on n'exclut pas la possibilité qu'un agent puisse avoir une position courte sur un marché.

(ii) Hypothèse d'accès inégal. Dans chaque pays, on suppose qu'il existe deux catégories d'investisseurs: les investisseurs restreints et les investisseurs non restreints (selon la terminologie de Errunza et Losq [1985], [1989]). Par définition, les investisseurs restreints n'ont pas accès à d'autre marché du capital que le leur. L'accès au marché étranger leur est totalement interdit (ou impossible ${ }^{1}$ ). Le seul actif risqué qu'ils peuvent

\footnotetext{
${ }^{1}$ La raison pour laquelle les investisseurs restreints ne peuvent investir à l'étranger n'est pas précisée. On a toutefois tendance à se référer implicitement à des barrières de type réglementaire, ce qui justifie
} 
détenir est celui émis sur leur propre marché. Les investisseurs non restreints, on l'aura compris, peuvent librement et sans contrainte se porter acquéreurs ou vendeurs des actifs risqués domestique et étranger. Ils ne souffrent d'aucune discrimination, et sont traités sur le marché étranger sur un pied d'égalité avec les investisseurs de ce pays. Bien évidemment, on supposera que les ventes et achats d'actifs financiers sont entièrement centralisés et qu'aucune transaction bilatérale n'est possible. Les investisseurs non restreints ne peuvent donc se porter comme intermédiaires auprès des investisseurs restreints. Signalons enfin que nous recourons à l'hypothèse d'agent représentatif: les populations des investisseurs restreints et non restreints se réduisent chacune à un seul agent (dans chaque pays). Notre modèle fait donc intervenir au total quatre types d'agents.

(iii) Absence d'habitat monétaire préféré. Les agents des deux pays partagent le même numéraire, ce qui est le moyen avoué d'éviter le traitement du risque de change. Ce postulat se justifie en recourant à l'hypothèse d'absence de risque réel de change. Cela revient à supposer que tous les agents, indépendemment de leur nationalité, utilisent comme numéraire le même bien (ou panier de biens) de consommation, que tous consomment à l'identique (hypothèse d'homogénéité des comportements de consommation).

(iv) Prêts et emprunts sans risque au même taux d'intérêt. On suppose l'existence d'un secteur bancaire international parfaitement intégré, auquel ont accès de façon égale les investisseurs des deux pays, qu'ils soient restreints ou non. Chaque investisseur peut procéder à des dépôts bancaires ou au contraire emprunter un montant quelconque, cela pour une rémunération certaine et indépendante de sa position. Il peut donc prêter ou emprunter au même taux sans risque, dont la valeur nous est fournie de façon totalement exogène.

(v) Préférences moyenne-variance. Le comportement des agents est modélisé selon le paradigme bien connu du modèle «moyenne-variance». Les agents sont supposés myopes, de sorte qu'ils ne s'intéressent qu'à l'optimisation de leur portefeuille sur la présente période. Plus précisément, chacun d'entre eux est doté d'une fonction d'utilité dépendant positivement de l'espérance de sa richesse de fin de période et négativement de la variance. On suppose que les agents restreint et non restreint d'un même pays ont un

l'expression d' «accès inégal », mais cela n'est nullement nécessaire. Il se peut que cette population d'investisseurs soit restreinte de fait et non de droit, à cause de problèmes informationnels, cognitifs, ou autres. 
comportement identique face au risque (leurs fonctions d'utilité sont identiques). La tolérance (relative) au risque des investisseurs est la même pour tous les investisseurs d'un même pays. Seuls des investisseurs de nationalités différentes peuvent alors se comporter différemment dans des conditions égales.

(vi) Anticipations homogènes. On suppose que les agents qui ont accès à un marché ont des anticipations identiques à son sujet, ce qui peut être rationalisé en supposant qu'ils ont des informations similaires. Notons que l'on ne suppose pas que tous les agents ont les mêmes anticipations à propos de tous les actifs : les anticipations des agents restreints vis-àvis de l'actif qui leur est inaccessible nous importent peu, puisqu'elles n'influencent pas les conditions d'équilibre des marchés.

\subsection{Notations}

Pour éviter toute confusion, nommons nos quatre types d'agents. L'agent 1 (3) désigne l'agent représentatif de la population des investisseurs restreints du pays A (B), les agents non restreints des pays A et B étant respectivement désignés comme les agents 2 et 4. Le tableau 1 ci-dessous récapitule ces conventions.

Tableau 1. Les agents

\begin{tabular}{|c|c|c|}
\hline Pays d'appartenance & Catégorie & Désignation \\
\hline A & Investisseur restreint & Agent 1 \\
A & Investisseur non resteint & Agent 2 \\
B & Investisseur restreint & Agent 3 \\
B & Investisseur non restreint & Agent 4 \\
\hline
\end{tabular}

Chacun de ces agents est muni d'une richesse initiale, que l'on note $\mathrm{W}_{\mathrm{i}}$ (richesse initiale de l'agent $\mathrm{i}, \forall \mathrm{i} \in(1,2,3,4))$, qu'il investit entièrement dans les différents actifs qui sont à sa disposition, escomptant de cet investissement qu'il lui procure la richesse aléatoire de fin de période $\widetilde{\mathrm{W}}_{\mathrm{i}}$. La richesse initiale de chaque agent se définit en proportion de la « richesse nationale» de son pays. On note $\mathrm{W}_{\mathrm{A}}$ la richesse nationale initiale du pays $\mathrm{A}$, c'est-à-dire la somme de $\mathrm{W}_{1}$ et de $\mathrm{W}_{2}$, et $\mathrm{W}_{\mathrm{B}}$ celle du pays $\mathrm{B}$, soit la somme de $\mathrm{W}_{3}$ et $\mathrm{W}_{4}$. On définit les paramètres $\mathrm{q}_{\mathrm{A}}$ et $\mathrm{q}_{\mathrm{B}}$, représentatifs des barrières d'accès à l'investissement international, comme suit : 


$$
\begin{aligned}
& \mathrm{W}_{1}=\left(1-\mathrm{q}_{\mathrm{A}}\right) \mathrm{W}_{\mathrm{A}} \\
& \mathrm{W}_{2}=\mathrm{q}_{\mathrm{A}} \mathrm{W}_{\mathrm{A}} \\
& \mathrm{W}_{3}=\left(1-\mathrm{q}_{\mathrm{B}}\right) \mathrm{W}_{\mathrm{B}} \\
& \mathrm{W}_{4}=\mathrm{q}_{\mathrm{B}} \mathrm{W}_{\mathrm{B}}
\end{aligned}
$$

Les paramètres $\mathrm{q}_{\mathrm{A}}$ et $\mathrm{q}_{\mathrm{B}}$ désignent la part de la richesse des investisseurs non restreints dans la richesse de tous les investisseurs des pays A et B respectivement. Plus $\mathrm{q}_{\mathrm{A}}$ et $\mathrm{q}_{\mathrm{B}}$ sont proches de zéro, plus les barrières d'accès sont importantes. Ces deux paramètres résument donc l'état de segmentation de notre hypothétique marché international des capitaux.

En donnant des valeurs particulières à ces paramètres, on obtient des situations très caractéristiques du marché international des capitaux. Ainsi la segmentation complète des marchés se résume à la condition $\mathrm{q}_{\mathrm{A}}=\mathrm{q}_{\mathrm{B}}=0$, c'est-à-dire par l'absence total d'échange d'actifs entre les deux pays, tous les investisseurs étant des investisseurs restreints. A l'inverse, la situation d'intégration complète s'obtient en posant $\mathrm{q}_{\mathrm{A}}=\mathrm{q}_{\mathrm{B}}=1$. Il n'y alors que des investisseurs non restreints, ce qui témoigne de l'absence de toute barrière à l'investissement international. Tous les investisseurs sont alors traités également sur tous les marchés, indépendemment de leur nationalité. La situation de segmentation douce de Errunza et Losq [1985] s'obtient en posant $\mathrm{q}_{\mathrm{A}}=1, \mathrm{q}_{\mathrm{B}}=0$ (ou en posant $\mathrm{q}_{\mathrm{A}}=0, \mathrm{q}_{\mathrm{B}}=1$ ). Cette situation se traduit par une importante asymétrie dans la position des investisseurs des deux pays, les investisseurs du pays A étant totalement libres d'investir là où bon leur semble, alors que ceux du pays B sont restreints à l'investissement dans les limites de leur marché national (pour ce qui est des actifs risqués). Nous traitons ci-dessous le cas général où $\mathrm{q}_{\mathrm{A}}$ et $\mathrm{q}_{\mathrm{B}}$ sont de valeurs quelconques dans le domaine $[0,1] \times[0,1]$, les cas particuliers se déduisant des résultats généraux.

On note $\mathrm{P}_{\mathrm{A}}\left(\mathrm{P}_{\mathrm{B}}\right)$ le prix initial de l'actif domestique du marché $\mathrm{A}(\mathrm{B})$. On suppose conventionnellement qu'il n'y a qu'une seule part de ces actifs, de sorte que $\mathrm{P}_{\mathrm{A}}$ et $\mathrm{P}_{\mathrm{B}}$ désignent aussi la capitalisation totale de chacun de ces marchés. $\mathrm{P}_{\mathrm{M}}$ désigne la somme de $\mathrm{P}_{\mathrm{A}}$ et $\mathrm{P}_{\mathrm{B}}$, soit la capitalisation internationale totale des deux marchés. Les prix aléatoires de fin de période (dividendes éventuels compris) des actifs $A$ et $B$, et leur somme, sont respectivement désignés par $\widetilde{\mathrm{P}}_{\mathrm{A}}, \widetilde{\mathrm{P}}_{\mathrm{B}}$ et $\widetilde{\mathrm{P}}_{\mathrm{M}}$. Les espérances de ces prix futurs, $\mathrm{E}\left(\widetilde{\mathrm{P}}_{\mathrm{A}}\right)$ et $\mathrm{E}\left(\widetilde{\mathrm{P}}_{\mathrm{B}}\right)$, prises conditionnellement à l'information dont disposent les investisseurs, 
leurs variances $\mathrm{V}\left(\widetilde{\mathrm{P}}_{\mathrm{A}}\right)$ et $\mathrm{V}\left(\widetilde{\mathrm{P}}_{\mathrm{B}}\right)$ et leur covariance $\operatorname{Cov}\left(\widetilde{\mathrm{P}}_{\mathrm{A}}, \widetilde{\mathrm{P}}_{\mathrm{B}}\right)$, caractérisent entièrement les anticipations des investisseurs. On supposera que $\operatorname{Cov}\left(\widetilde{\mathrm{P}}_{\mathrm{A}}, \widetilde{\mathrm{P}}_{\mathrm{B}}\right)^{2} \neq \mathrm{V}\left(\widetilde{\mathrm{P}}_{\mathrm{A}}\right) \mathrm{V}\left(\widetilde{\mathrm{P}}_{\mathrm{B}}\right)$.

Le modèle que nous présentons s'exprime alternativement en termes de prix ou en termes de rentabilités. Nous notons $\widetilde{\mathrm{R}}_{\mathrm{A}}=\frac{\widetilde{\mathrm{P}}_{\mathrm{A}}}{\mathrm{P}_{\mathrm{A}}}$ la rentabilité aléatoire de l'actif A sur la période considérée, $\widetilde{R}_{B}=\frac{\widetilde{P}_{B}}{P_{B}}$ celle de l'actif $B$, et $\widetilde{R}_{M}=\frac{\widetilde{P}_{M}}{P_{M}}$ celle du portefeuille de marché international. On pose que la rémunération d'un dépôt sans risque ou d'un emprunt de même nature auprès du secteur bancaire se fait au taux d'intérêt sans risque R-1.

Le choix de portefeuille de l'agent $i$ est représenté par le triplet $\left(D_{i}, X_{A i}, X_{B i}\right)$, où $D_{i}$ désigne le montant de son dépôt bancaire sans risque, et $\mathrm{X}_{\mathrm{Ai}}\left(\mathrm{X}_{\mathrm{Bi}}\right)$ le nombre de parts de l'actif A (B) dont il se porte acquéreur. Avec ces définitions la contrainte budgétaire de l'agent $\mathrm{i}$ est facile à définir :

$$
\mathrm{D}_{\mathrm{i}}+\mathrm{X}_{\mathrm{Ai}} \mathrm{P}_{\mathrm{A}}+\mathrm{X}_{\mathrm{Bi}} \mathrm{P}_{\mathrm{B}}=\mathrm{W}_{\mathrm{i}}
$$

La connaissance du choix de portefeuille de l'agent i conduit par ailleurs à définir sa richesse aléatoire de fin de période :

$$
\widetilde{\mathrm{W}}_{\mathrm{i}}=\mathrm{D}_{\mathrm{i}} \mathrm{R}+\mathrm{X}_{\mathrm{Ai}} \widetilde{\mathrm{P}}_{\mathrm{A}}+\mathrm{X}_{\mathrm{Bi}} \widetilde{\mathrm{P}}_{\mathrm{B}},
$$

que l'on peut exprimer, à l'aide de la contrainte budgétaire, en fonction du seul choix de portefeuille des actifs risqués, soit en fonction du couple $\left(\mathrm{X}_{\mathrm{Ai}}, \mathrm{X}_{\mathrm{Bi}}\right)$ :

$$
\widetilde{\mathrm{W}}_{\mathrm{i}}=\mathrm{W}_{\mathrm{i}} \mathrm{R}+\mathrm{X}_{\mathrm{Ai}}\left(\widetilde{\mathrm{P}}_{\mathrm{A}}-\mathrm{P}_{\mathrm{A}} \mathrm{R}\right)+\mathrm{X}_{\mathrm{Bi}}\left(\widetilde{\mathrm{P}}_{\mathrm{B}}-\mathrm{P}_{\mathrm{B}} \mathrm{R}\right)
$$

Nous supposons que les investisseurs sont des optimisateurs moyenne-variance. L'agent i est donc doté d'une fonction d'utilité $U_{i}$ présentant les caractéristiques suivantes :

$$
\mathrm{U}_{\mathrm{i}}=\mathrm{U}_{\mathrm{i}}\left[\mathrm{E}\left(\widetilde{\mathrm{W}}_{\mathrm{i}}\right), \mathrm{V}\left(\widetilde{\mathrm{W}}_{\mathrm{i}}\right)\right], \operatorname{avec} \frac{\partial \mathrm{U}_{\mathrm{i}}}{\partial \mathrm{E}\left(\widetilde{\mathrm{W}}_{\mathrm{i}}\right)}>0, \frac{\partial \mathrm{U}_{\mathrm{i}}}{\partial \mathrm{V}\left(\widetilde{\mathrm{W}}_{\mathrm{i}}\right)}<0
$$

La première dérivée stipule que l'investisseur est d'autant plus satisfait que sa richesse future attendue est grande; la seconde traduit son aversion pour le risque, représenté ici par la variance de sa richesse finale. Pour rendre notre modèle plus maniable, il nous faut paramétriser plus précisément cette fonction d'utilité. A cet effet, nous supposerons que la tolérance au risque de l'investisseur i obéit à la relation : 


$$
-\frac{\partial U_{i} / \partial E\left(\widetilde{W}_{i}\right)}{2 \partial U_{i} / \partial V\left(\widetilde{W}_{i}\right)}=\rho_{i} W_{i},
$$

où $\rho_{\mathrm{i}}$ est une constante, qui représente la tolérance au risque de l'investisseur i. Comme les équations de demande d'actifs risqués dérivées plus loin en témoignent, l'équation (08) stipule que l'agent i a une aversion relative au risque constante. En d'autres termes, la proportion d'actifs risqués qu'il demande est proportionnelle à sa richesse.

Nous faisons l'hypothèse que les investisseurs restreints et non restreints d'un même pays ont une fonction d'utilité identique. Cela se traduit par l'hypothèse d'égalité de leur coefficient de tolérance au risque :

$$
\rho_{1}=\rho_{2}=\rho_{\text {A }} \text { et } \rho_{3}=\rho_{4}=\rho_{B}
$$

Les hypothèses et les notations de notre modèle étant clairement précisées, on peut à présent entreprendre sa résolution.

\section{L’équilibre du marché des capitaux}

\subsection{Les choix de portefeuille optimaux}

Dans une première étape, il convient d'étudier les choix de portefeuille de nos différents agents. Considérons pour commencer les choix de portefeuille des individus non restreints, soit pour $i \in\{2,4\}$. L'individu non restreint $i$, libre d'investir où il veut, maximise sa fonction d'utilité à travers le choix de $X_{\mathrm{Ai}}$ et $\mathrm{X}_{\mathrm{Bi}}$, qui sont les deux quantités sous son contrôle. Son choix de portefeuille optimal vérifie la condition de premier ordre :

$$
\left\{\begin{array}{l}
\frac{\partial \mathrm{U}_{\mathrm{i}}\left[\mathrm{E}\left(\widetilde{\mathrm{W}}_{\mathrm{i}}\right), \mathrm{V}\left(\widetilde{\mathrm{W}}_{\mathrm{i}}\right)\right]}{\partial \mathrm{X}_{\mathrm{Ai}}}=0 \\
\frac{\partial \mathrm{U}_{\mathrm{i}}\left[\mathrm{E}\left(\widetilde{\mathrm{W}}_{\mathrm{i}}\right), \mathrm{V}\left(\widetilde{\mathrm{W}}_{\mathrm{i}}\right)\right]}{\partial \mathrm{X}_{\mathrm{Bi}}}=0
\end{array}\right.
$$

Les calculs conduisent à un système de deux équations à deux inconnues que l'on résout pour obtenir le choix de portefeuille optimal de l'agent i :

$\forall \mathrm{i} \in(2,4)$, les commandes optimales $\mathrm{X}_{\mathrm{Ai}}^{*}$ et $\mathrm{X}_{\mathrm{Bi}}^{*}$ sont :

$$
\mathrm{X}_{\mathrm{Ai}}^{*}=\frac{\rho_{\mathrm{i}} \mathrm{W}_{\mathrm{i}}}{\mathrm{V}\left(\widetilde{\mathrm{P}}_{\mathrm{A}}\right) \mathrm{V}\left(\widetilde{\mathrm{P}}_{\mathrm{B}}\right)-\operatorname{Cov}\left(\widetilde{\mathrm{P}}_{\mathrm{A}}, \widetilde{\mathrm{P}}_{\mathrm{B}}\right)^{2}}\left(\mathrm{~V}\left(\widetilde{\mathrm{P}}_{\mathrm{B}}\right)\left[\mathrm{E}\left(\widetilde{\mathrm{P}}_{\mathrm{A}}\right)-\mathrm{P}_{\mathrm{A}} \mathrm{R}\right]-\operatorname{Cov}\left(\widetilde{\mathrm{P}}_{\mathrm{A}}, \widetilde{\mathrm{P}}_{\mathrm{B}}\right)\left[\mathrm{E}\left(\widetilde{\mathrm{P}}_{\mathrm{B}}\right)-\mathrm{P}_{\mathrm{B}} \mathrm{R}\right]\right)(
$$




$$
X_{B i}^{*}=\frac{\rho_{i} W_{i}}{V\left(\widetilde{P}_{A}\right) V\left(\widetilde{P}_{B}\right)-\operatorname{Cov}\left(\widetilde{P}_{A}, \widetilde{P}_{B}\right)^{2}}\left(V\left(\widetilde{P}_{A}\right)\left[E\left(\widetilde{P}_{B}\right)-P_{B} R\right]-\operatorname{Cov}\left(\widetilde{P}_{A}, \widetilde{P}_{B}\right)\left[E\left(\widetilde{P}_{A}\right)-P_{A} R\right]\right)(
$$

Le nombre de parts d'actifs risqués que l'agent demande est proportionnel à sa richesse, et est d'autant plus important qu'il est tolérant au risque. Le choix de portefeuille optimal donné par (11) et (12) concerne les agents non restreints des deux pays. Bien que de nationalités différentes, les agents 2 et 4 font somme toute des choix similaires, qui ne diffèrent que par l'importance de leur tolérance au risque et de leur richesse initiale. Ce résultat est tout à fait logique puisque ce sont finalement les deux seules caractéristiques qui distinguent ces deux types d'agents.

Pour ce qui est des agents restreints, leurs choix optimaux sont encore plus simples à dériver. Considérons pour commencer l'agent restreint du pays A (l'agent 1). Par définition, il ne peut accéder au marché $B$, de sorte que sa commande d'actif étranger est nécessairement nulle : $\mathrm{X}_{\mathrm{B} 1}^{*}=0$. L'agent $1 \mathrm{n}$ 'a à sa discrétion que le choix de $\mathrm{X}_{\mathrm{A} 1}$. De la condition du premier ordre :

$$
\frac{\partial \mathrm{U}_{1}\left[\mathrm{E}\left(\widetilde{\mathrm{W}}_{1}\right), \mathrm{V}\left(\widetilde{\mathrm{W}}_{1}\right)\right]}{\partial \mathrm{X}_{\mathrm{A} 1}}=0,
$$

on tire le choix optimal de l'agent B :

$$
X_{A 1}^{*}=\rho_{1} W_{1} \frac{E\left(\widetilde{P}_{A}\right)-P_{A} R}{V\left(\widetilde{P}_{A}\right)}
$$

De façon tout à fait symétrique, on peut dériver le choix de portefeuille optimal de l'agent restreint du pays B (l'agent 3). Ne pouvant accéder au marché A, sa demande optimale d'actif $\mathrm{A} \quad \mathrm{X}_{\mathrm{A} 3}^{*}$ est nécessairement nulle, et sa demande de l'actif domestique est donnée par :

$$
X_{B 3}^{*}=\rho_{3} W_{3} \frac{E\left(\widetilde{P}_{B}\right)-P_{B} R}{V\left(\widetilde{P}_{B}\right)}
$$

Les fonctions de demande des différents investisseurs étant déterminées, la prochaine étape de la résolution du modèle consiste à les agréger pour obtenir les fonctions de demande globale des actifs risqués. Notons $X_{A G}$ et $X_{B G}$ ces fonctions de demande globale. Elles se définissent comme suit :

$$
\mathrm{X}_{\mathrm{AG}}=\mathrm{X}_{\mathrm{A} 1}^{*}+\mathrm{X}_{\mathrm{A} 2}^{*}+\mathrm{X}_{\mathrm{A} 4}^{*} \text { et } \mathrm{X}_{\mathrm{BG}}=\mathrm{X}_{\mathrm{B} 2}^{*}+\mathrm{X}_{\mathrm{B} 3}^{*}+\mathrm{X}_{\mathrm{B} 4}^{*} \text {, }
$$

soit en intégrant les équations de demande optimales des différents investisseurs : 


$$
\begin{aligned}
X_{A G} & =\frac{\left(\rho_{A} q_{A} W_{A}+\rho_{B} q_{B} W_{B}\right)}{V\left(\widetilde{P}_{A}\right) V\left(\widetilde{P}_{B}\right)-\operatorname{Cov}\left(\widetilde{P}_{A}, \widetilde{P}_{B}\right)^{2}}\left(V\left(\widetilde{P}_{B}\right)\left[E\left(\widetilde{P}_{A}\right)-P_{A} R\right]-\operatorname{Cov}\left(\widetilde{P}_{A}, \widetilde{P}_{B}\right)\left[E\left(\widetilde{P}_{B}\right)-P_{B} R\right]\right) \\
& +\rho_{A}\left(1-q_{A}\right) W_{A} \frac{\left[E\left(\widetilde{P}_{A}\right)-P_{A} R\right]}{V\left(\widetilde{P}_{A}\right)} \\
X_{B G} & =\frac{\left(\rho_{A} q_{A} W_{A}+\rho_{B} q_{B} W_{B}\right)}{V\left(\widetilde{P}_{A}\right) V\left(\widetilde{P}_{B}\right)-\operatorname{Cov}\left(\widetilde{P}_{A}, \widetilde{P}_{B}\right)^{2}}\left(V\left(\widetilde{P}_{A}\right)\left[E\left(\widetilde{P}_{B}\right)-P_{B} R\right]-\operatorname{Cov}\left(\widetilde{P}_{A}, \widetilde{P}_{B}\right)\left[E\left(\widetilde{P}_{A}\right)-P_{A} R\right]\right) \\
& +\rho_{B}\left(1-q_{B}\right) W_{B} \frac{\left[E\left(\widetilde{P}_{B}\right)-P_{B} R\right]}{V\left(\widetilde{P}_{B}\right)}
\end{aligned}
$$

\subsection{L'équilibre du marché international du capital}

L'offre de chaque actif est conventionnellement fixée à 1, puisque nous avons supposé qu'il n'y en avait qu'une seule part. Par définition, le vecteur de prix d'équilibre $\left(\mathrm{P}_{\mathrm{A}}^{*}, \mathrm{P}_{\mathrm{B}}^{*}\right)$ est donc solution du système d'équations :

$$
\left\{\begin{array}{l}
\mathrm{X}_{\mathrm{AG}}\left(\mathrm{P}_{\mathrm{A}}^{*}, \mathrm{P}_{\mathrm{B}}^{*}\right)=1 \\
\mathrm{X}_{\mathrm{BG}}\left(\mathrm{P}_{\mathrm{A}}^{*}, \mathrm{P}_{\mathrm{B}}^{*}\right)=1
\end{array}\right.
$$

La résolution de ce système conduit à :

$$
\begin{aligned}
& P_{A}^{*}=\gamma_{A}\left(q_{A}, q_{B}\right)+\frac{E\left(\widetilde{P}_{A}\right)}{R}-\frac{\operatorname{Cov}\left(\widetilde{P}_{A}, \widetilde{P}_{M}\right)}{\left(\rho_{A} W_{A}+\rho_{B} W_{B}\right) R} \\
& P_{B}^{*}=\gamma_{B}\left(q_{A}, q_{B}\right)+\frac{E\left(\widetilde{P}_{B}\right)}{R}-\frac{\operatorname{Cov}\left(\widetilde{P}_{B}, \widetilde{P}_{M}\right)}{\left(\rho_{A} W_{A}+\rho_{B} W_{B}\right) R}
\end{aligned}
$$

où $\gamma_{A}\left(q_{A}, q_{B}\right)$ et $\gamma_{B}\left(q_{A}, q_{B}\right)$ sont définis par :

$$
\begin{aligned}
\gamma_{A}\left(q_{A}, q_{B}\right)= & \frac{\operatorname{Cov}\left(\widetilde{P}_{A}, \widetilde{P}_{M}\right)}{\left(\rho_{A} W_{A}+\rho_{B} W_{B}\right) R}-\frac{\left(q_{A} \rho_{A} W_{A}+q_{B} \rho_{B} W_{B}\right) \operatorname{Cov}\left(\widetilde{P}_{A}, \widetilde{P}_{M}\right)}{\left[V\left(\widetilde{P}_{A}\right) V\left(\widetilde{P}_{B}\right)-\operatorname{Cov}\left(\widetilde{P}_{A}, \widetilde{P}_{B}\right)^{2}\right] \Gamma\left(q_{A}, q_{B}\right) R} \\
& -\frac{\left(1-q_{B}\right) \rho_{B} W_{B}}{V\left(\widetilde{P}_{B}\right) \Gamma\left(q_{A}, q_{B}\right) R} \\
\gamma_{B}\left(q_{A}, q_{B}\right)= & \frac{\operatorname{Cov}\left(\widetilde{P}_{B}, \widetilde{P}_{M}\right)}{\left(\rho_{A} W_{A}+\rho_{B} W_{B}\right) R}-\frac{\left(q_{A} \rho_{A} W_{A}+q_{B} \rho_{B} W_{B}\right) \operatorname{Cov}\left(\widetilde{P}_{B}, \widetilde{P}_{M}\right)}{\left[V\left(\widetilde{P}_{A}\right) V\left(\widetilde{P}_{B}\right)-\operatorname{Cov}\left(\widetilde{P}_{A}, \widetilde{P}_{B}\right)^{2}\right] \Gamma\left(q_{A}, q_{B}\right) R} \\
& -\frac{\left(1-q_{A}\right) \rho_{A} W_{A}}{V\left(\widetilde{P}_{A}\right) \Gamma\left(q_{A}, q_{B}\right) R},
\end{aligned}
$$

avec $\Gamma\left(\mathrm{q}_{\mathrm{A}}, \mathrm{q}_{\mathrm{B}}\right)$ défini par :

$\Gamma\left(q_{A}, q_{B}\right)=\frac{\left(q_{A} \rho_{A} W_{A}+q_{B} \rho_{B} W_{B}\right)}{V\left(\widetilde{P}_{A}\right) V\left(\widetilde{P}_{B}\right)-\operatorname{Cov}\left(\widetilde{P}_{A}, \widetilde{P}_{B}\right)^{2}}\left(\rho_{A} W_{A}+\rho_{B} W_{B}\right)+\frac{\left(1-q_{A}\right)\left(1-q_{B}\right) \rho_{A} W_{A} \rho_{B} W_{B}}{V\left(\widetilde{P}_{A}\right) V\left(\widetilde{P}_{B}\right)}$ 
Les équations (20) et (21) donnent les prix d'équilibre des actifs risqués A et B pour des proportions quelconques d'investisseurs non restreints, et constituent les équations générales de notre modèle. Plutôt que d'exprimer ces équations d'équilibre en termes de prix, on peut indifféremment les exprimer en termes de rentabilité :

$$
\begin{aligned}
& \mathrm{E}\left(\widetilde{\mathrm{R}}_{\mathrm{A}}\right)-\mathrm{R}=\alpha_{\mathrm{A}}\left(\mathrm{q}_{\mathrm{A}}, \mathrm{q}_{\mathrm{B}}\right)+\lambda \operatorname{Cov}\left(\widetilde{\mathrm{R}}_{\mathrm{A}}, \widetilde{\mathrm{R}}_{\mathrm{M}}\right) \\
& \mathrm{E}\left(\widetilde{\mathrm{R}}_{\mathrm{B}}\right)-\mathrm{R}=\alpha_{\mathrm{B}}\left(\mathrm{q}_{\mathrm{A}}, \mathrm{q}_{\mathrm{B}}\right)+\lambda \operatorname{Cov}\left(\widetilde{\mathrm{R}}_{\mathrm{B}}, \widetilde{\mathrm{R}}_{\mathrm{M}}\right),
\end{aligned}
$$

où $\alpha_{A}\left(q_{A}, q_{B}\right)$ et $\alpha_{B}\left(q_{A}, q_{B}\right)$ sont définis par :

$$
\begin{aligned}
& \alpha_{A}\left(q_{A}, q_{B}\right)=\frac{\left(1-q_{B}\right) \rho_{B} W_{B}}{\Gamma\left(q_{A}, q_{B}\right) V\left(\widetilde{P}_{B}\right) P_{A}^{*}}+\left[\frac{\left(q_{A} \rho_{A} W_{A}+q_{B} \rho_{B} W_{B}\right) P_{M}^{*}}{\left[V\left(\widetilde{P}_{A}\right) V\left(\widetilde{P}_{B}\right)-\operatorname{Cov}\left(\widetilde{P}_{A}, \widetilde{P}_{B}\right)^{2}\right] \Gamma\left(q_{A}, q_{B}\right)}-\lambda\right] \operatorname{Cov}\left(\widetilde{R}_{A}, \widetilde{R}_{M}\right) \\
& \alpha_{B}\left(q_{A}, q_{B}\right)=\frac{\left(1-q_{A}\right) \rho_{A} W_{A}}{\Gamma\left(q_{A}, q_{B}\right) V\left(\widetilde{P}_{A}\right) P_{B}^{*}}+\left[\frac{\left(q_{A} \rho_{A} W_{A}+q_{B} \rho_{B} W_{B}\right) P_{M}^{*}}{\left[V\left(\widetilde{P}_{A}\right) V\left(\widetilde{P}_{B}\right)-\operatorname{Cov}\left(\widetilde{P}_{A}, \widetilde{P}_{B}\right)^{2}\right] \Gamma\left(q_{A}, q_{B}\right)}-\lambda\right] \operatorname{Cov}\left(\widetilde{R}_{B}, \widetilde{R}_{M}\right)
\end{aligned}
$$

Ces équations ne sont pas d'interprétation facile dans le cas général où $\mathrm{q}_{\mathrm{A}}$ et $\mathrm{q}_{\mathrm{B}}$ sont de valeurs quelconques. Nous considérons ci-dessous les cas particuliers que constituent les cas de segmentation complète, d'intégration complète et de segmentation douce.

D'après la représentation des barrières à l'investissement international que donne notre modèle, les marchés de capitaux A et B sont complètement segmentés lorsqu'il n'y a dans chaque pays que des investisseurs restreints : $\mathrm{q}_{\mathrm{A}}=\mathrm{q}_{\mathrm{B}}=0$. Les équations d'équilibre de prix et de rentabilité se simplifient aisément lorsqu'on introduit cette contrainte.

Le cas de marchés segmentés : $\mathrm{q}_{\mathrm{A}}=\mathrm{q}_{\mathrm{B}}=0$

$$
\begin{aligned}
& P_{A}^{*}=\frac{E\left(\widetilde{P}_{A}\right)}{R}-\frac{V\left(\widetilde{P}_{A}\right)}{\left(\rho_{A} W_{A}\right) R} \Leftrightarrow E\left(\widetilde{R}_{A}\right)-R=\frac{P_{A}^{*}}{\rho_{A} W_{A}} V\left(\widetilde{R}_{A}\right) \\
& P_{B}^{*}=\frac{E\left(\widetilde{P}_{B}\right)}{R}-\frac{V\left(\widetilde{P}_{B}\right)}{\left(\rho_{B} W_{B}\right) R} \Leftrightarrow E\left(\widetilde{R}_{B}\right)-R=\frac{P_{B}^{*}}{\rho_{B} W_{B}} V\left(\widetilde{R}_{B}\right)
\end{aligned}
$$

Dans le cas de marchés segmentés, on retrouve les équations d'équilibre du CAPM domestique de Sharpe [1964], Lintner [1965] et Mossin [1966]. L'excès de rentabilité de chaque portefeuille de marché national est proportionnel à la variance de sa rentabilité. Seul le risque purement domestique est rémunéré. Et cette rémunération, à des prix $\mathrm{P}_{\mathrm{A}}^{*} / \rho_{\mathrm{A}} \mathrm{W}_{\mathrm{A}}$ pour l'actif $\mathrm{A}$ et $\mathrm{P}_{\mathrm{B}}^{*} / \rho_{\mathrm{B}} \mathrm{W}_{\mathrm{B}}$ pour l'actif $\mathrm{B}$, est elle aussi fixée sur une base entièrement domestique. 
Le deuxième cas à considérer est celui de marchés complètement intégrés. Ne sont alors présents cette fois-ci sur les marchés que des investisseurs non restreints : $\mathrm{q}_{\mathrm{A}}=\mathrm{q}_{\mathrm{B}}=1$.

Le cas de marchés intégrés : $\mathrm{q}_{\mathrm{A}}=\mathrm{q}_{\mathrm{B}}=1$

$$
\begin{aligned}
& P_{A}^{*}=\frac{E\left(\widetilde{P}_{A}\right)}{R}-\frac{\operatorname{Cov}\left(\widetilde{P}_{A}, \widetilde{P}_{M}\right)}{\left(\rho_{A} W_{A}+\rho_{B} W_{B}\right) R} \Leftrightarrow E\left(\widetilde{R}_{A}\right)-R=\lambda \operatorname{Cov}\left(\widetilde{R}_{A}, \widetilde{R}_{M}\right) \\
& P_{B}^{*}=\frac{E\left(\widetilde{P}_{B}\right)}{R}-\frac{\operatorname{Cov}\left(\widetilde{P}_{B}, \widetilde{P}_{M}\right)}{\left(\rho_{A} W_{A}+\rho_{B} W_{B}\right) R} \Leftrightarrow E\left(\widetilde{R}_{B}\right)-R=\lambda \operatorname{Cov}\left(\widetilde{R}_{B}, \widetilde{R}_{M}\right)
\end{aligned}
$$

où $\lambda=\frac{P_{M}^{*}}{\left(\rho_{A} W_{A}+\rho_{B} W_{B}\right)}$.

Dans le cas de marchés intégrés, on retrouve le CAPM international, qui diffère du CAPM domestique par le remplacement du benchmark domestique par le porteuille de marché international. Cette fois-ci, l'excès de rentabilité de chaque actif est proportionnel à sa covariance avec la rentabilité du portefeuille de marché international. La prime de risque $\lambda$, fixée sur une base internationale, est cette fois-ci commune aux deux marchés. C'est cette dernière caractéristique qui reflète la parfaite cohérence des deux marchés ou la loi du prix unique du risque.

La dernière situation particulière que nous considèrerons est celle de segmentation douce développée par Errunza et Losq [1985]. Dans cette situation les investisseurs d'un des deux marchés, que nous poserons être le marché A, sont tous non restreints, et ceux de l'autre marché sont tous des investisseurs restreints : $\mathrm{q}_{\mathrm{A}}=1, \mathrm{q}_{\mathrm{B}}=0$.

Le cas de segmentation douce : $\mathrm{q}_{\mathrm{A}}=1, \mathrm{q}_{\mathrm{B}}=0$

$$
\begin{aligned}
& P_{A}^{*}=\frac{E\left(\widetilde{P}_{A}\right)}{R}-\frac{\operatorname{Cov}\left(\widetilde{P}_{A}, \widetilde{P}_{M}\right)}{\left(\rho_{A} W_{A}+\rho_{B} W_{B}\right) R}-\frac{\rho_{B} W_{B}\left[V\left(\widetilde{P}_{A}\right) V\left(\widetilde{P}_{B}\right)-\operatorname{Cov}\left(\widetilde{P}_{A}, \widetilde{P}_{B}\right)^{2}\right]}{\rho_{A} W_{A} V\left(\widetilde{P}_{B}\right)\left(\rho_{A} W_{A}+\rho_{B} W_{B}\right) R} \\
& \Leftrightarrow E\left(\widetilde{R}_{A}\right)-R=\lambda \operatorname{Cov}\left(\widetilde{R}_{A}, \widetilde{R}_{M}\right)+\frac{\rho_{B} W_{B}\left[V\left(\widetilde{P}_{A}\right) V\left(\widetilde{P}_{B}\right)-\operatorname{Cov}\left(\widetilde{P}_{A}, \widetilde{P}_{B}\right)^{2}\right]}{\rho_{A} W_{A} V\left(\widetilde{P}_{B}\right)\left(\rho_{A} W_{A}+\rho_{B} W_{B}\right) P_{A}^{*}} \\
& P_{B}^{*}=\frac{E\left(\widetilde{P}_{B}\right)}{R}-\frac{\operatorname{Cov}\left(\widetilde{P}_{A}, \widetilde{P}_{M}\right)}{\left(\rho_{A} W_{A}+\rho_{B} W_{B}\right) R} \Leftrightarrow E\left(\widetilde{R}_{B}\right)-R=\lambda \operatorname{Cov}\left(\widetilde{R}_{B}, \widetilde{R}_{M}\right)
\end{aligned}
$$

On retrouve les résultats de Errunza et Losq [1985], à savoir que l'actif B qui est l'actif «éligible» selon leur terminologie, c'est-à-dire accessible à tous les investisseurs indépendemment de leur nationalité, offre une rentabilité attendue et se paie au même prix 
que si le marché était intégré. La segmentation douce d'un marché n'affecte donc pas l'actif « éligible ». Pour ce qui est de l'actif « inéligible », l'actif A, inaccessible aux investisseurs du pays $\mathrm{B}$, son prix est inférieur à celui qui prévaudrait si le marché était intégré, et en conséquence offre une rémunération attendue supérieure. L'équation de rentabilité de l'actif A fait apparaître une «super prime de risque » (Errunza et Losq [1985]) par rapport à la situation d'intégration, de valeur $\frac{\rho_{\mathrm{B}} \mathrm{W}_{\mathrm{B}}\left[\mathrm{V}\left(\widetilde{\mathrm{P}}_{\mathrm{A}}\right) \mathrm{V}\left(\widetilde{\mathrm{P}}_{\mathrm{B}}\right)-\operatorname{Cov}\left(\widetilde{\mathrm{P}}_{\mathrm{A}}, \widetilde{\mathrm{P}}_{\mathrm{B}}\right)^{2}\right]}{\rho_{\mathrm{A}} \mathrm{W}_{\mathrm{A}} \mathrm{V}\left(\widetilde{\mathrm{P}}_{\mathrm{B}}\right)\left(\rho_{\mathrm{A}} \mathrm{W}_{\mathrm{A}}+\rho_{\mathrm{B}} \mathrm{W}_{\mathrm{B}}\right) \mathrm{P}_{\mathrm{A}}^{*}}$.

La comparaison des situations de segmentation douce et d'intégration défend l'idée que l'ouverture d'un marché conduit à son appréciation. En témoigne le prix de l'actif A inférieur en situation de segmentation douce au prix affiché en situation d'intégration. Ce résultat s'obtient cependant en considérant une libéralisation « univariée », puisque l'un des deux marchés (le marché B) est déjà à l'origine complètement ouvert aux investisseurs étrangers. L'examen ci-dessous du cas général d'une libéralisation simultanée des deux marchés conduit au désaccord avec Errunza et Losq [1985].

\section{Ecarts de pricing et erreurs d'évaluation}

\subsection{Définitions}

Revenons au modèle général, qui établit les équations de prix pour des valeurs quelconques de $\mathrm{q}_{\mathrm{A}}$ et $\mathrm{q}_{\mathrm{B}}$ (équations (20) et (21)). Si l'on compare ces équations avec celles tirées du cas particulier de marchés intégrés (équations (31) et (32)), alors on observe que $\gamma_{A}\left(q_{A}, q_{B}\right)$ et $\gamma_{B}\left(q_{A}, q_{B}\right)$ désignent les différences entre les prix affichés des actifs et ceux caractéristiques d'un marché intégré. Nous appellerons écarts de pricing ces quantités (en référence à la «norme » définie par l'intégration des marchés). De la même façon, les termes $\alpha_{A}\left(q_{A}, q_{B}\right)$ et $\alpha_{B}\left(q_{A}, q_{B}\right)$, que nous appelerons erreurs d'évaluation, désignent les différentiels de rentabilité des actifs par rapport à la rentabilité «normale» de marchés intégrés.

La comparaison des écarts de pricing et des erreurs d'évaluation conduit à remarquer qu'elles sont liées par les relations :

$$
\gamma_{\mathrm{k}}\left(\mathrm{q}_{\mathrm{A}}, \mathrm{q}_{\mathrm{B}}\right)=-\frac{\mathrm{P}_{\mathrm{k}}^{*}}{\mathrm{R}} \alpha_{\mathrm{k}}\left(\mathrm{q}_{\mathrm{A}}, \mathrm{q}_{\mathrm{B}}\right), \mathrm{k} \in\{\mathrm{A}, \mathrm{B}\}
$$


L'écart de pricing est de signe opposé à celui de l'erreur d'évaluation, ce qui est cohérent. Si un actif manifeste une erreur d'évaluation positive, c'est-à-dire si sa rémunération est supérieure à ce qu'elle devrait être par rapport à la situation de marchés intégrés, c'est bien parce que cet actif se paie à un prix moindre (écart de pricing négatif) que celui qui devrait être le sien.

Les écarts de pricing sont nuls lorsque les erreurs d'évaluation le sont aussi, et vice versa, les unes étant liées aux autres par une relation de proportion. Cette caractéristique fait que l'on peut indifféremment étudier l'effet de l'atténuation des barrières à l'investissement international sur les uns ou les autres.

\subsection{Les effets de la libéralisation sur les prix}

Le modèle de Errunza et Losq [1985] nous enseigne, en comparant la situation de segmentation douce avec celle d'intégration, que la libéralisation des marchés conduit nécessairement à une hausse des prix des actifs concernés. Le cadre plus général développé ici permet de contester cette assertion. Quel effet a généralement une hausse de $\mathrm{q}_{\mathrm{A}}$ et $\mathrm{q}_{\mathrm{B}}$ sur $\mathrm{P}_{\mathrm{A}}^{*}$ et $\mathrm{P}_{\mathrm{B}}^{*}$ ? Le traitement du problème pour les deux marchés étant symétrique, tenons-nous en à l'étude d'un seul actif, par exemple l'actif $\mathrm{A}$. Par définition la différentielle totale de $\mathrm{P}_{\mathrm{A}}^{*}$ vaut :

$$
\mathrm{dP}_{\mathrm{A}}^{*}=\frac{\partial \mathrm{P}_{\mathrm{A}}^{*}}{\partial \mathrm{q}_{\mathrm{A}}} \mathrm{dq}_{\mathrm{A}}+\frac{\partial \mathrm{P}_{\mathrm{A}}^{*}}{\partial \mathrm{q}_{\mathrm{B}}} \mathrm{dq}_{\mathrm{B}}=\frac{\partial \gamma_{\mathrm{A}}}{\partial \mathrm{q}_{\mathrm{A}}} \mathrm{dq}_{\mathrm{A}}+\frac{\partial \gamma_{\mathrm{A}}}{\partial \mathrm{q}_{\mathrm{B}}} \mathrm{dq}_{\mathrm{B}}
$$

avec :

$$
\begin{aligned}
\frac{\partial \gamma_{A}\left(q_{A}, q_{B}\right)}{\partial q_{A}}= & -\frac{\operatorname{Cov}\left(\widetilde{P}_{A}, \widetilde{P}_{M}\right)\left[\rho_{A} W_{A} \Gamma\left(q_{A}, q_{B}\right)-\frac{\partial \Gamma\left(q_{A}, q_{B}\right)}{\partial q_{A}}\left(q_{A} \rho_{A} W_{A}+q_{B} \rho_{B} W_{B}\right)\right]}{\left[V\left(\widetilde{P}_{A}\right) V\left(\widetilde{P}_{B}\right)-\operatorname{Cov}\left(\widetilde{P}_{A}, \widetilde{P}_{B}\right)^{2}\right] \Gamma\left(q_{A}, q_{B}\right)^{2} R} \\
& +\frac{\left(1-q_{B}\right) \rho_{B} W_{B} \frac{\partial \Gamma\left(q_{A}, q_{B}\right)}{\partial q_{A}}}{V\left(\widetilde{P}_{B}\right) \Gamma\left(q_{A}, q_{B}\right)^{2} R}
\end{aligned}
$$




$$
\begin{aligned}
\frac{\partial \gamma_{A}\left(q_{A}, q_{B}\right)}{\partial q_{B}}= & -\frac{\operatorname{Cov}\left(\widetilde{P}_{A}, \widetilde{P}_{M}\right)\left[\rho_{B} W_{B} \Gamma\left(q_{A}, q_{B}\right)-\frac{\partial \Gamma\left(q_{A}, q_{B}\right)}{\partial q_{B}}\left(q_{A} \rho_{A} W_{A}+q_{B} \rho_{B} W_{B}\right)\right]}{\left[V\left(\widetilde{P}_{A}\right) V\left(\widetilde{P}_{B}\right)-\operatorname{Cov}\left(\widetilde{P}_{A}, \widetilde{P}_{B}\right)^{2}\right] \Gamma\left(q_{A}, q_{B}\right)^{2} R} \\
& +\frac{\rho_{B} W_{B} \Gamma\left(q_{A}, q_{B}\right)+\left(1-q_{B}\right) \rho_{B} W_{B} \frac{\partial \Gamma\left(q_{A}, q_{B}\right)}{\partial q_{B}}}{V\left(\widetilde{P}_{B}\right) \Gamma\left(q_{A}, q_{B}\right)^{2} R},
\end{aligned}
$$

où les dérivées partielles $\frac{\partial \Gamma\left(\mathrm{q}_{\mathrm{A}}, \mathrm{q}_{\mathrm{B}}\right)}{\partial \mathrm{q}_{\mathrm{A}}}$ et $\frac{\partial \Gamma\left(\mathrm{q}_{\mathrm{A}}, \mathrm{q}_{\mathrm{B}}\right)}{\partial \mathrm{q}_{\mathrm{B}}}$ valent :

$$
\begin{aligned}
& \frac{\partial \Gamma\left(q_{A}, q_{B}\right)}{\partial q_{A}}=\frac{\rho_{A} W_{A}\left(\rho_{A} W_{A}+\rho_{B} W_{B}\right)}{V\left(\widetilde{P}_{A}\right) V\left(\widetilde{P}_{B}\right)-\operatorname{Cov}\left(\widetilde{P}_{A}, \widetilde{P}_{B}\right)^{2}}-\frac{\left(1-q_{B}\right)\left(\rho_{A} W_{A}\right)\left(\rho_{B} W_{B}\right)}{V\left(\widetilde{P}_{A}\right) V\left(\widetilde{P}_{B}\right)} \\
& \frac{\partial \Gamma\left(q_{A}, q_{B}\right)}{\partial q_{B}}=\frac{\rho_{B} W_{B}\left(\rho_{A} W_{A}+\rho_{B} W_{B}\right)}{V\left(\widetilde{P}_{A}\right) V\left(\widetilde{P}_{B}\right)-\operatorname{Cov}\left(\widetilde{P}_{A}, \widetilde{P}_{B}\right)^{2}}-\frac{\left(1-q_{A}\right)\left(\rho_{A} W_{A}\right)\left(\rho_{B} W_{B}\right)}{V\left(\widetilde{P}_{A}\right) V\left(\widetilde{P}_{B}\right)}
\end{aligned}
$$

Si l'on réintègre (37) et (38) dans (36), on obtient une équation assez lourde. De façon générale on se contentera d'observer qu'il est impossible de «signer» cette différentielle totale. On ne peut donc absolument pas affirmer que la libéralisation des marchés provoque inévitablement une appréciation du prix des actifs.

Ce n'est que dans certains cas particuliers que le signe de cette différentielle est connu. Considérons par exemple l'effet de la libéralisation sur le prix de l'actif A en situation de segmentation douce. Dans une telle situation $\left(\mathrm{q}_{\mathrm{A}}=1, \mathrm{q}_{\mathrm{B}}=0\right)$, la globalisation ne peut venir que de la hausse de $\mathrm{q}_{\mathrm{B}}, \mathrm{q}_{\mathrm{A}}$ ayant déjà atteint sa valeur maximale sur $[0,1]$. En posant $\mathrm{dq}_{\mathrm{A}}=0$, on obtient que $\mathrm{dP}_{\mathrm{A}}^{*}$ prend la valeur particulière au point de segmentation douce :

$$
\mathrm{dP}_{\mathrm{A}}^{*}\left(\mathrm{q}_{\mathrm{A}}=1, \mathrm{q}_{\mathrm{B}}=0\right)=\frac{\left(\rho_{\mathrm{B}} \mathrm{W}_{\mathrm{B}}\right)\left[\mathrm{V}\left(\widetilde{\mathrm{P}}_{\mathrm{A}}\right) \mathrm{V}\left(\widetilde{\mathrm{P}}_{\mathrm{B}}\right)-\operatorname{Cov}\left(\widetilde{\mathrm{P}}_{\mathrm{A}}, \widetilde{\mathrm{P}}_{\mathrm{B}}\right)^{2}\right]}{\left(\rho_{\mathrm{A}} \mathrm{W}_{\mathrm{A}}\right)^{2} \mathrm{~V}\left(\widetilde{\mathrm{P}}_{\mathrm{B}}\right) \mathrm{R}} \mathrm{dq}_{\mathrm{B}}>0 \quad\left(\operatorname{pour} \mathrm{dq}_{\mathrm{B}}>0\right)
$$

Dans ce cas particulier, la différentielle est positive. Un accroissement du nombre d'investisseurs étrangers qui accèdent au marché A provoque un accroissement du prix de l'actif A. Ce résultat particulier n'est cependant pas généralisable à toutes les situations.

Dans le cas général développé plus haut, la différentielle du prix d'équilibre de l'actif A est une fonction non linéaire des paramètres $\mathrm{q}_{\mathrm{A}}$ et $\mathrm{q}_{\mathrm{B}}$, et il n'est pas possible de la «signer» pour des valeurs positives quelconques de $\mathrm{dq}_{\mathrm{A}}$ et $\mathrm{dq}_{\mathrm{B}}$. D'abord, le signe de chaque dérivée partielle $\frac{\partial \mathrm{P}_{\mathrm{A}}^{*}}{\partial \mathrm{q}_{\mathrm{A}}}$ et $\frac{\partial \mathrm{P}_{\mathrm{A}}^{*}}{\partial \mathrm{q}_{\mathrm{B}}}$ est inconnu. A priori, on attendrait plutôt de la 
première dérivée qu'elle soit négative et de la seconde qu'elle soit positive. Ainsi, on attendrait d'une augmentation de la population des investisseurs non restreints du pays A (relativement à celle des investisseurs restreints) qu'elle s'accompagne d'une hausse de la demande d'actifs étrangers, au détriment des actifs domestiques, déprimant ainsi le prix de l'actif domestique. Inversement, on attendrait plutôt d'une hausse de la population des investisseurs non restreints du pays étranger une augmentation de la demande de l'actif domestique, et une hausse consécutive de son prix. Malheureusement, ces raisonnements reposent sur l'idée que les investisseurs non restreints demandent plus d'actifs étrangers que les investisseurs restreints du même pays, ce qui n'est pas nécessairement vrai. En raison de la possibilité de vente à découvert, il se peut effectivement que la position optimale des investisseurs non restreints à l'étranger soit une position courte : il n'est pas impossible qu'il soit optimal pour eux de vendre à découvert des actifs étrangers pour acheter encore plus d'actifs domestiques. Dans ce cas, les investisseurs non restreints forment des demandes d'actifs étrangers inférieures (car négatives) à celles des investisseurs restreints, ce qui explique que les dérivées ci-dessus puissent ne pas présenter les signes attendus.

Ecartons ce genre de possibilités. Donnons des valeurs «raisonnables» aux paramètres de notre économie, de sorte que les positions des investisseurs soient toutes des positions longues. Dans ce cas, les dérivées ci-dessus sont respectivement négatives et positives $\frac{\partial \mathrm{P}_{\mathrm{A}}^{*}}{\partial \mathrm{q}_{\mathrm{A}}}<0, \frac{\partial \mathrm{P}_{\mathrm{A}}^{*}}{\partial \mathrm{q}_{\mathrm{B}}}>0$. Mais même ainsi, l'effet du processus de globalisation sur les prix des actifs demeure ambigu, en raison du caractère bivarié de ce processus.

Considérons l'exemple numérique de deux marchés aux caractéristiques suivantes : $\rho_{\mathrm{A}} \mathrm{W}_{\mathrm{A}}=\rho_{\mathrm{B}} \mathrm{W}_{\mathrm{B}}=\rho \mathrm{W}, \mathrm{V}\left(\widetilde{\mathrm{P}}_{\mathrm{A}}\right)=\mathrm{V}\left(\widetilde{\mathrm{P}}_{\mathrm{B}}\right)=\sigma^{2}, \operatorname{Cov}\left(\widetilde{\mathrm{P}}_{\mathrm{A}}, \widetilde{\mathrm{P}}_{\mathrm{B}}\right)=0,5 \sigma^{4}, \mathrm{q}_{\mathrm{A}}=\mathrm{q}_{\mathrm{B}}=0,5$

Sous ces conditions, on a :

$$
\mathrm{dP}_{\mathrm{A}}^{*}=-\frac{12 \sigma^{2}}{245(\rho \mathrm{W}) \mathrm{R}} \mathrm{dq}_{\mathrm{A}}+\frac{72 \sigma^{2}}{245(\rho \mathrm{W}) \mathrm{R}} \mathrm{dq}_{\mathrm{B}}
$$

Le signe de cette différentielle dépend de la variation relative de $\mathrm{q}_{\mathrm{A}}$ et de $\mathrm{q}_{\mathrm{B}}$. Ces deux variations ayant des effets qui s'opposent, toutes les configurations sont possibles. L'équation (43) confirme d'abord les conclusions du cas classique d'une libéralisation « univariée ». Si on pose $\mathrm{dq}_{\mathrm{A}}=0$, on obtient que $\mathrm{dP}_{\mathrm{A}}^{*}=\frac{72 \sigma^{2}}{245(\rho \mathrm{W}) \mathrm{R}} \mathrm{dq}_{\mathrm{B}}>0$ (pour 
$\left.\mathrm{dq}_{\mathrm{B}}>0\right)$. La libéralisation univariée du marché $\mathrm{A}$ conduit à une appréciation $\mathrm{du}$ prix de l'actif A. Plus généralement, la différentielle totale gardera ce même signe positif tant que la condition $\mathrm{dq}_{\mathrm{B}}>6 \mathrm{dq}_{\mathrm{A}}$ sera respectée. La libéralisation simultanée des deux marchés peut aussi avoir des effets inattendus. Ainsi, dans le cas très particulier où l'on l'égalité $\mathrm{dq}_{\mathrm{B}}=6 \mathrm{dq}_{\mathrm{A}}$, les effets sur $\mathrm{P}_{\mathrm{A}}^{*}$ des deux libéralisations se compensent de sorte que le prix est inchangé. L'atténuation des barrières à l'investissement international n'a alors aucun effet sur le prix d'équilibre de l'actif $\mathrm{A}$. Certes, l'apparition de ce phénomène de compensation nécessite des conditions assez strictes, il n'en demeure pas moins qu'elle est possible. Enfin, dans le cas où $\mathrm{dq}_{\mathrm{B}}<6 \mathrm{dq}_{\mathrm{A}}$, la libéralisation simultanée des deux marchés produit un effet absolument inverse à celui que laissent escompter les analyses classiques. Dans ce cas, l'ouverture du marché A produit un effet positif sur le prix de l'actif domestique, mais cet effet est plus que compensé par l'ouverture plus importante de l'autre marché.

Notre analyse des effets de la libéralisation sur les prix des actifs montre que la prise en compte de la simultanéité des processus de libéralisation des marchés conduit à tempérer les résultats des analyses classiques. Cette simple constatation permet de répondre à l'énigme soulevée par Stulz [1999] : si la plupart des études empiriques sur les effets de la libéralisation font état d'effets assez faibles, c'est sans doute parce que les processus de libéralisation des différents marchés ont lieu plus ou moins en même temps, et qu'ils se concurrencent finalement les uns les autres, les effets des uns étant en conséquence plus ou moins compensés par ceux des autres.

\subsection{Libéralisation et intégration des marchés de capitaux}

Les écarts de pricing et les erreurs d'évaluation tendent-elles à se résorber avec l'augmentation de $\mathrm{q}_{\mathrm{A}}$ et $\mathrm{q}_{\mathrm{B}}$, c'est-à-dire avec la levée des restrictions sur les investisseurs? C'est à cette question que nous tentons de répondre dans ce paragraphe. Habituellement on considère d'après un raisonnement purement intuitif que moins de barrières vaut mieux que plus en termes de cohérence des marchés de capitaux. La disparition progressive des barrières internationales serait favorable à la disparition progressive des écarts de pricing et des erreurs d'évaluation des actifs. De façon tout à fait concomittante avec les résultats du paragraphe précédent, nous allons voir que la libéralisation des marchés ne conduit pas 
nécessairement à une plus grande intégration, la simultanéité des processus de libéralisation étant à nouveau en cause.

Dans notre modèle, ces barrières sont d'autant moins importantes que $\mathrm{q}_{\mathrm{A}}$ et $\mathrm{q}_{\mathrm{B}}$ sont grands (proches de 1). Raisonnons en termes d'écarts de pricing. Ces derniers peuvent être négatifs comme positifs. C'est donc leur importance en valeur absolue qui nous intéresse. Tenons-nous en à nouveau à l'étude d'un seul actif $\mathrm{A}$, l'actif $\mathrm{A}$ par exemple, le traitement de l'actif B étant tout à fait similaire. Nous dirons de l'écart de pricing de l'actif A qu'elle tend à se résorber avec l'augmentation de $\mathrm{q}_{\mathrm{A}}$ et $\mathrm{q}_{\mathrm{B}} \mathrm{si}\left|\gamma_{\mathrm{A}}\left(\mathrm{q}_{\mathrm{A}}, \mathrm{q}_{\mathrm{B}}\right)\right|$ diminue. Le problème de la fonction $\left|\gamma_{A}\left(q_{A}, q_{B}\right)\right|$ est qu'elle n'est pas dérivable en tout point. On lui préfère pour cette raison la fonction $\gamma_{A}\left(q_{A}, q_{B}\right)^{2}$, qui en constitue une transformation monotone. La différentielle totale de cette fonction vaut :

$d \gamma_{A}\left(q_{A}, q_{B}\right)^{2}=2 \gamma_{A}\left(q_{A}, q_{B}\right) \frac{\partial \gamma_{A}\left(q_{A}, q_{B}\right)}{\partial q_{A}} d_{A}+2 \gamma_{A}\left(q_{A}, q_{B}\right) \frac{\partial \gamma_{A}\left(q_{A}, q_{B}\right)}{\partial q_{B}} d_{q_{B}}(44)$

De façon générale, il est impossible de signer cette différentielle totale, pour une variation donnée de $\mathrm{q}_{\mathrm{A}}$ et $\mathrm{q}_{\mathrm{B}}$. On ne peut absolument pas déduire d'une variation à la hausse de $\mathrm{q}_{\mathrm{A}}$ et $\mathrm{q}_{\mathrm{B}}$ qu'elle conduit nécessairement à une baisse de $\gamma_{\mathrm{A}}\left(\mathrm{q}_{\mathrm{A}}, \mathrm{q}_{\mathrm{B}}\right)^{2}$. Des résultats tout à fait similaires sont obtenus si l'on raisonne en termes d'erreurs d'évaluation plutôt qu'en termes d'écarts de pricing, les différentielles de ces deux quantités étant liées par la relation

$$
d \alpha_{A}\left(q_{A}, q_{B}\right)^{2}=\frac{R^{2}\left[\frac{E\left(\widetilde{P}_{A}\right)}{R}-\frac{\operatorname{Cov}\left(\widetilde{P}_{A}, \widetilde{P}_{M}\right)}{\left(\rho_{A} W_{A}+\rho_{B} W_{B}\right) R}\right]}{\left(P_{A}^{*}\right)^{3}} d \gamma_{A}\left(q_{A}, q_{B}\right)^{2}
$$

L'erreur d'évaluation diminue (en valeur absolue) si l'écart de pricing diminue luimême, et vice versa. On se concentrera donc sur l'étude d'une seule de ces différentielles, celle de l'écart de pricing. L'étude générale de cette différentielle est assez complexe car elle est une fonction non linéaire d'assez nombreux paramètres. Son signe étant généralement indeterminé, on ne peut en déduire que la libéralisation a pour effet inévitable la réduction des écarts de pricing. Une libéralisation accrue des marchés de capitaux ne conduit donc pas obligatoirement à une plus grande intégration. 
La libéralisation des marchés de capitaux peut donc conduire à une diminution ou bien à une augmentation des erreurs de pricing (et des rentabilités anormales), ou encore ne rien y changer. On retrouve des ambiguités pareilles à celles du paragraphe précédent, qui ne peuvent être levées que dans quelques cas très particuliers. Reprenons par exemple le cas particulier de segmentation douce $\left(\mathrm{q}_{\mathrm{A}}=1, \mathrm{q}_{\mathrm{B}}=0\right)$. Dans une telle situation, on rappelle que la globalisation ne peut venir que de la hausse de $\mathrm{q}_{\mathrm{B}}, \mathrm{q}_{\mathrm{A}}$ étant déjà à sa valeur maximale. Le processus de libéralisation envisagé est donc caractérisé par $\mathrm{dq}_{\mathrm{A}}=0, \mathrm{dq}_{\mathrm{B}}>0$. On impose $\mathrm{dq}_{\mathrm{A}}=0$, et on calcule la valeur de $\mathrm{d} \gamma_{\mathrm{A}}\left(\mathrm{q}_{\mathrm{A}}, \mathrm{q}_{\mathrm{B}}\right)^{2}$ au point $\left(\mathrm{q}_{\mathrm{A}}=1, \mathrm{q}_{\mathrm{B}}=0\right)$ :

$$
\mathrm{d} \gamma_{\mathrm{A}}\left(\mathrm{q}_{\mathrm{A}}=1, \mathrm{q}_{\mathrm{B}}=0\right)^{2}=2 \gamma_{\mathrm{A}}\left(\mathrm{q}_{\mathrm{A}}=1, \mathrm{q}_{\mathrm{B}}=0\right) \frac{\left(\rho_{\mathrm{B}} \mathrm{W}_{\mathrm{B}}\right)\left[\mathrm{V}\left(\widetilde{\mathrm{P}}_{\mathrm{A}}\right) \mathrm{V}\left(\widetilde{\mathrm{P}}_{\mathrm{B}}\right)-\operatorname{Cov}\left(\widetilde{\mathrm{P}}_{\mathrm{A}}, \widetilde{\mathrm{P}}_{\mathrm{B}}\right)^{2}\right]}{\left(\rho_{\mathrm{A}} \mathrm{W}_{\mathrm{A}}\right)^{2} \mathrm{~V}\left(\widetilde{\mathrm{P}}_{\mathrm{B}}\right) \mathrm{R}}
$$

Au point $\left(\mathrm{q}_{\mathrm{A}}=1, \mathrm{q}_{\mathrm{B}}=0\right)$ l'écart de pricing de l'actif $\mathrm{A}$ est négatif :

$$
\gamma_{A}\left(q_{A}=1, q_{B}=0\right)=-\frac{\left(\rho_{B} W_{B}\right)\left[V\left(\widetilde{P}_{A}\right) V\left(\widetilde{P}_{B}\right)-\operatorname{Cov}\left(\widetilde{P}_{A}, \widetilde{P}_{B}\right)^{2}\right]}{\left(\rho_{A} W_{A}\right) V\left(\widetilde{P}_{B}\right)\left(\rho_{A} W_{A}+\rho_{B} W_{B}\right) R}<0
$$

En tenant compte de résultat, on parvient à «signer » la différentielle de l'écart de pricing (élevé au carré) :

$$
\mathrm{d} \gamma_{\mathrm{A}}\left(\mathrm{q}_{\mathrm{A}}=1, \mathrm{q}_{\mathrm{B}}=0\right)^{2}<0
$$

Dans le cas particulier de la segmentation douce, on parvient à montrer qu'ouvrir le marché A permet de réduire la distorsion de prix relevée sur l'actif domestique. La libéralisation de ce marché conduit alors à une plus grande intégration financière. Les résultats de ce cas particulier ne s'étendent cependant pas au cas général.

Pour illustrer ce point, on peut reprendre l'exemple numérique du paragraphe précédent. Sous ces conditions, l'écart de pricing de l'actif A vaut :

$$
\gamma_{A}\left(q_{A}, q_{B}\right)=-\frac{3 \sigma^{2}}{28(\rho W) R}<0
$$

L'actif A est sous-évalué. Il s'échange à un prix moindre que celui qui prévaudrait dans un marché intégré. Comment une hausse de $\mathrm{q}_{\mathrm{A}}$ et $\mathrm{q}_{\mathrm{B}}$ agit-elle sur cette erreur de pricing? Calculons sa différentielle totale :

$$
\mathrm{d} \gamma_{\mathrm{A}}\left(\mathrm{q}_{\mathrm{A}}, \mathrm{q}_{\mathrm{B}}\right)^{2}=\frac{18}{1715}\left[\frac{\sigma^{2}}{(\sigma \mathrm{W}) \mathrm{R}}\right]^{2} \mathrm{dq}_{\mathrm{A}}-\frac{108}{1715}\left[\frac{\sigma^{2}}{(\rho \mathrm{W}) \mathrm{R}}\right]^{2} \mathrm{dq}_{\mathrm{B}}
$$


L'examen de cette différentielle est similaire à celui de la différentielle du prix d'équilibre fait au paragraphe précédent. On observe que son signe dépend de la variation relative de $\mathrm{q}_{\mathrm{A}}$ et $\mathrm{q}_{\mathrm{B}}$. A nouveau, diverses configurations sont possibles, selon le signe de $d q_{B}-6 d q_{A}$. Il se peut d'abord que les effets des libéralisations des deux marchés se compensent. Cela surviendra si $\mathrm{dq}_{\mathrm{B}}=6 \mathrm{dq}_{\mathrm{A}}$. La différentielle totale est alors nulle, l'atténuation des barrières à l'investissement international n'ayant dans ce cas aucun effet sur l'écart de pricing de l'actif $A$. La différentielle totale sera négative si $\mathrm{dq}_{\mathrm{B}}>6 \mathrm{dq}_{\mathrm{A}}$. Dans ce cas l'écart de pricing se résorbe. L'effet contraire sera observé à chaque fois que l'on aura $\mathrm{dq}_{\mathrm{B}}<6 \mathrm{dq}_{\mathrm{A}}$.

A travers cet exemple on voit bien que l'on ne peut absolument pas conclure de la libéralisation qu'elle conduit nécessairement à faire converger les prix des actifs vers les prix qui seraient les leurs si les marchés étaient intégrés. Les écarts vis-à-vis de la loi du prix unique du risque peuvent tout à fait exhiber des évolutions inattendues ou non monotones $\mathrm{au}$ fur et à mesure que les barrières sont levées. Une libéralisation plus grande n'est pas nécessairement la preuve d'une plus grande intégration des marchés de capitaux.

\section{Conclusion}

Indéniablement, les barrières à l'investissement international se sont progressivement atténuées au cours des deux dernières décennies. Pourtant, les marchés de capitaux ne sont pas encore complètement globalisés, contrairement au sentiment populaire. Beaucoup de barrières à l'investissement, certaines moins visibles que d'autres, se dressent encore entre les marchés et les investisseurs ${ }^{1}$. «En dépit de l'accélération des flux de capitaux transfrontaliers durant les années 90, l'internationalisation des marchés de capitaux est encore dans l'enfance » (Tesar [1999]). Le home bias observé dans les portefeuilles des investisseurs demeure en effet substantiel. Au début des années 80, 2\% du portefeuille d'actions des américains était détenu sous forme d'actifs étrangers, contre $12 \%$ à la fin des années 90 (Tesar [1999]). Certains modèles d'évaluation conduisent à estimer que la part théorique de titres étrangers dans les portefeuilles des américains devrait être de l'ordre de

\footnotetext{
${ }^{1}$ A ce sujet il faut bien garder à l'esprit qu'une barrière levée d'un point de vue règlementaire ne s'en trouve pas pour autant abolie obligatoirement dans les faits, et qu'elle peut donc continuer à exercer plus ou moins
} 
$50 \%$. A la vitesse où le home bias se résorbe depuis vingt ans, cela signifie que la correction restante nécessiterait encore quelques décennies.

La poursuite du processus de globalisation ou de libéralisation sera-t-elle source d'inflation du prix des actifs financiers ? Doit-on en attendre que les distorsions de prix et de rentabilité produites par les barrières restantes s'atténuent toujours progressivement? A ces deux questions, et contrairement à l'idée communément admise, notre analyse nous porte à répondre par la négative. La disparition progressive des barrières à l'investissement international n'a pas un effet univoque sur les prix, et on ne peut nullement assurer que la libéralisation progressive des marchés de capitaux conduise à redonner peu à peu leur cohérence aux marchés de capitaux en termes de pricing, les acheminant de façon monotone vers la loi du prix unique.

tous ses effets. L'explication de ce phénomène tient à la crédibilité des politiques de libéralisation des marchés. 


\section{REFERENCES}

ADLER M. et DUMAS B. [1983], « International Portfolio Choice and Corporation Finance: A Synthesis », Journal of Finance, 38, 925-984.

ALEXANDER G.J., EUN C.S. et JANAKIRAMANAN S. [1987], « Asset Pricing and Dual Listing on Foreign Capital Markets : A Note », Journal of Finance, 42, 151-158.

ALFORD A. [1993], "Assessing Capital Market Segmentation: A Review of the Literature ", in S.R. STANSELL (éd.), International Financial Market Integration, Basil Blackwell, Cambridge.

BASAK S. [1996], «An Intertemporal Model of International Capital Market Segmentation », Journal of Financial and Quantitative Analysis, 31, 161-188.

BEKAERT G. et HARVEY C. [2000], «Foreign Speculators and Emerging Equity Markets », Journal of Finance, 55, 565-613.

BLACK F. [1972], " Capital Market Equilibrium with Restricted Borrowing », Journal of Business, 45, 444-455.

BLACK F. [1974], « International Capital Market Equilibrium with Investment Barriers », Journal of Financial Economics, 1, 337-352.

BORDES C. [1988], «Interprétation théorique du mouvement d'intégration des marchés de capitaux », Cahiers Economiques et Monétaires, 31, 5-47.

CHEN Z. et KNEZ P.J. [1995], " Measurement of Market Integration and Arbitrage », Review of Financial Studies, 8, 287-325.

DOUKAS J. et SWITZER L.N. [2000], « Common Stock Returns and International Listing Announcements : Conditional Tests of the mild Segmentation Hypothesis », Journal of Banking and Finance, 24, 471-502.

ERRUNZA V. et LOSQ E. [1985], « International Asset Pricing under Mild Segmentation: Theory and Test », Journal of Finance, 40, 105-124.

ERRUNZA V. et LOSQ E. [1989], « Capital Flow Controls, International Asset Pricing, and Investors' Welfare: A Multi-Country Framework », Journal of Finance, 44, 10251037.

EUN C.S. et JANAKIRAMANAN S. [1986], «A Model of International Asset Pricing with a Constraint on the Foreign Equity Ownership », Journal of Finance, 41, 897-914.

FOERSTER S.R. et KAROLYI G.A. [1999], « The Effects of Market Segmentation and Investor Recognition on Asset Prices : Evidence from Foreign Stocks Listing in the United States », Journal of Finance, 54, 981-1013.

GOYEAU D., LEONARD J. et PEPIN D. [1999], «L'intégration internationale des marchés financiers: analyse et étude empirique », Economie Appliquée, 52, 93-115.

HENRY P.B. [2000], « Stock Market Liberalization, Economic Reform, and Emerging Market Equity Prices », Journal of Finance, 55, 529-564.

HIETALA P.T. [1989], « Asset Pricing in Partially Segmented Markets: Evidence from the Finnish Market », Journal of Finance, 44, 697-718.

KIM E.H. et SINGAL V. [2000], «Stock Market Openings : Experience of Emerging Economies », Journal of Business, 73, 25-66.

LEWIS K.K. [1999], «Trying to Explain Home Bias in Equities and Consumption », Journal of Economic Literature, 37, 571-608. 
LINTNER J. [1965], " The Valuation of Risk Assets and the Selection of Risky Investments in Stock Portfolios and Capital Budgets ", Review of Economics and Statistics, 47, 13-37.

LUCAS R.E. [1978], « Asset Prices in an Exchange Economy », Econometrica, 46, 14291145.

MERTON R.C. [1973], « An Intertemporal Capital Asset Pricing Model », Econometrica, 41, 867-887.

MOSSIN J. [1966], « Equilibrium in a Capital Asset Market », Econometrica, 34, 768-783.

ROSS S.A. [1976], " The Arbitrage Theory of Capital Asset Pricing ", Journal of Economic Theory, 13, 341-360.

SHARPE W.F. [1964], " Capital Asset Prices: A Theory of Market Equilibrium under Conditions of Risk », Journal of Finance, 19, 425-442.

SOLNIK B.H. [1974], "An Equilibrium Model of the International Capital Market », Journal of Economic Theory, 8, 500-524.

SOLNIK B.H. [1983], « International Arbitrage Pricing Theory », Journal of Finance, 38, 449-457.

STULZ R.M. [1981a], « A Model of International Asset Pricing », Journal of Financial Economics, 9, 383-406.

STULZ R.M. [1981b], « On the Effects of Barriers to International Investment », Journal of Finance, 36, 923-934.

STULZ R.M. [1995], «International Portfolio Choice and Asset Pricing: An Integrative Survey », in R.A. JARROW, V. MAKSIMOVIC et W.T. ZIEMBA (éd.), Finance, Elsevier Science B.V., Amsterdam.

STULZ R.M. [1999], « Globalization of Equity Markets and the Cost of Capital », NYSE Working Paper.

TESAR L.L [1999], « The Rôle of Equity in International Capital Flows », in M. FELDSTEIN (ed), International Capital Flows, University of Chicago Press, Chicago.

WHEATLEY S.M. [1988], " Some Tests of International Equity Integration », Journal of Financial Economics, 21, 177-212. 